\title{
Rectovaginal Fistula after Low Anterior Resection for Rectal Cancer Using a Double Stapling Technique
}

\author{
Satoshi Yodonawa Isao Ogawa Susumu Yoshida \\ Hiromichi Ito Keisuke Kobayashi Ryoko Kubokawa \\ Department of Surgery, Ibaraki Seinan Medical Center Hospital, Ibaraki, Japan
}

\section{Key Words}

Rectovaginal fistula - Double stapling · Rectal cancer - Transverse colostomy ·

Transvaginal repair

\begin{abstract}
A 55-year-old female underwent low anterior resection for rectal cancer using a double stapling technique. She developed a rectovaginal fistula on the 9th postoperative day. She was discharged from hospital after undergoing transverse colostomy, and 5 months later she underwent transvaginal repair of the rectovaginal fistula. She subsequently had an uneventful recovery. The leading cause of this complication is involvement of the posterior wall of the vagina in the staple line when firing the circular stapler. Transvaginal repair with a diverting stoma for rectovaginal fistula is a safe, minimally invasive and effective method.
\end{abstract}

\section{Introduction}

Coloanal or low colorectal anastomosis using the double stapling technique (DST) has become common in low anterior resection for rectal cancer $[1,2]$. However, this method occasionally results in rectovaginal fistula (RVF), with a reported incidence of RVF after low anterior resection of $0.9-2.9 \%$ [2-4]. Here, we report a case of RVF after low anterior resection for rectal cancer using DST, in whom successful repair was performed using a transvaginal approach with diverting colostomy.

\section{Case Report}

A 55-year-old woman was admitted to our hospital with acute colonic obstruction due to rectal cancer, which was confirmed by colonoscopy. Subsequently, an endoscopic decompression procedure 
using a transanal ileus tube was attempted. The lower edge of the cancer was $7 \mathrm{~cm}$ from the anal verge. Low anterior resection was performed following adequate cleaning of the colon. The rectal stump was closed with a linear stapler $2 \mathrm{~cm}$ below the cancer. A circular stapler gun (CDH $29 \mathrm{~mm}$, Ethicon Endosurgery, Cincinnati, Ohio, USA) was introduced through the middle of the stapled anal stump, mated with the anvil, and fired. The patient was stable postoperatively, but she noticed discharge of stool from the vagina on the 9th postoperative day. Vaginography revealed a RVF just above the level of anastomosis (fig. 1), and transverse colostomy was performed on the next day under the diagnosis of RVF. One month later, colonoscopy revealed that the fistula was $3 \mathrm{~cm}$ from the dentate line, with a well-circumscribed $1 \mathrm{~cm}$ hole through the rectal vaginal septum and many staple pins around the hole (fig. 2). The RVF had not closed spontaneously 4 months later, and a decision was made to repair the fistula.

Approximately 5 months postoperatively, a transvaginal approach was used to repair the fistula with simple closure of the vaginal and rectal wall. Preoperative mechanical preparation for this procedure was not required due to use of a diverting colostomy. Under general anesthesia, the patient was placed in the lithotomy position and the RVF was identified. A solution of adrenalin in saline $(1: 200,000)$ was injected under the posterior vaginal mucosa and a transvaginal incision was made longitudinally around the RVF. The edges of the incision were undermined circumferentially just over the external rectal mucosal layer. The fistula was probed and debrided. There were many staple pins around the fistula, and these were removed. The rectal opening of the fistula was then closed longitudinally with a single layer of absorbable sutures. Since the fistula was slightly high, it was not possible to interpose the levator ani muscles between the vaginal and rectal wall, and so the vaginal wall was finally closed with a single layer over the rectal wall.

The patient had a benign postoperative course and was discharged on the 5th postoperative day. Approximately 3 months postoperatively, colonoscopy and barium enema examination revealed that the RVF had completely healed without recurrence. The diverting loop colostomy was closed 5 months later and the patient has since had no sign or symptoms of rectal disease.

\section{Discussion}

Coloanal or low colorectal anastomosis using DST is used widely in low anterior resection for rectal cancer [1,2], but may cause RVF formation. The incidence of this complication is probably correlated with the experience and skill of the surgeon using the stapler. The leading cause of RVF is involvement of the posterior wall of the vagina in the staple line when firing the circular stapler due to poor visualization of the operative field in the deep pelvis $[5,6]$. In describing potential errors in DST, Sugarbaker suggested that if the rectal stump and posterior vagina have not been separated sufficiently and the staple gun is angled too far anteriorly, the posterior vagina may be compressed by the anvil and staple gun and partially resected by the circular knife [6]. To prevent this complication, the rectum should be dissected free of the posterior wall of the vagina and the staple gun should be angled more posteriorly to keep the vagina out of the circular anastomosis [6]. Additionally, it is important to check the vagina carefully by digital examination before firing the circular stapler.

Once a fistula occurs, management must be individualized. Various surgical procedures have been proposed for RVF repair after low anterior resection $[3,4,7]$, but management is difficult and outcomes are often unsatisfactory. Rex and Khubchandani reported that simple diverting colostomy resulted in a low rate of successful spontaneous healing of 35.3\% (6 of 17 patients), whereas endoanal repair gave higher rates of $62.5 \%$ ( 5 of 8 patients) in staged repair with diversion and 66.7\% ( 2 of 3 patients) in repair without diversion, and reanastomosis gave a rate of successful healing of 100\% (6 of 6 patients) [3].

We decided to try the transvaginal approach after diverting colostomy because this procedure is minimally invasive and has been successful in several previous reports $[4,7]$. We believe that the success of this procedure depends on the use of diverting colostomy 
before the transvaginal operation. Fecal diversion has several benefits, including diversion of the fecal stream away from the healing fistula, which decreases local inflammation and improves healing of perianal lesions. Additionally, no fecal inflammation occurs if the repair fails, and repeated repair can be performed. The transvaginal approach also offers numerous advantages, but this procedure must be performed with gentle dissection using careful hemostasis, full mobilization, accurate appositioning of healthy tissue without tension, adequate vascularization, and precise excision of the fistula tract followed by a meticulously layered closure.

Fig. 1. Vaginography revealed a RVF just above the level of anastomosis.

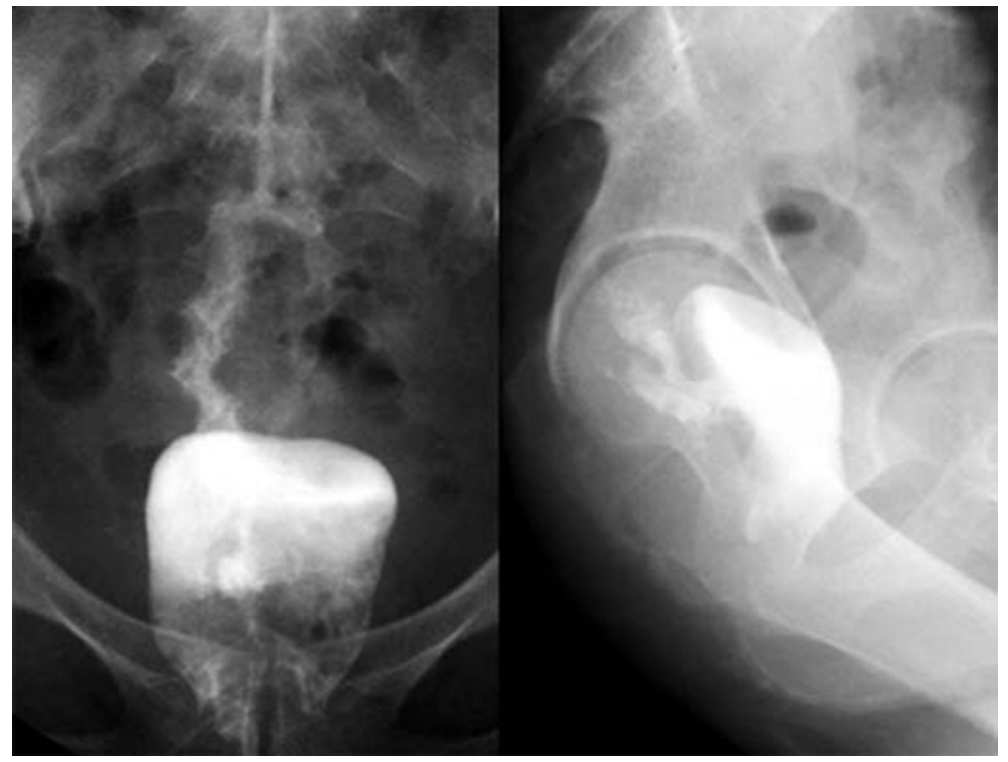


Fig. 2. Colonoscopy revealed a well-circumscribed $1 \mathrm{~cm}$ hole through the rectal vaginal septum and many staple pins around the hole.

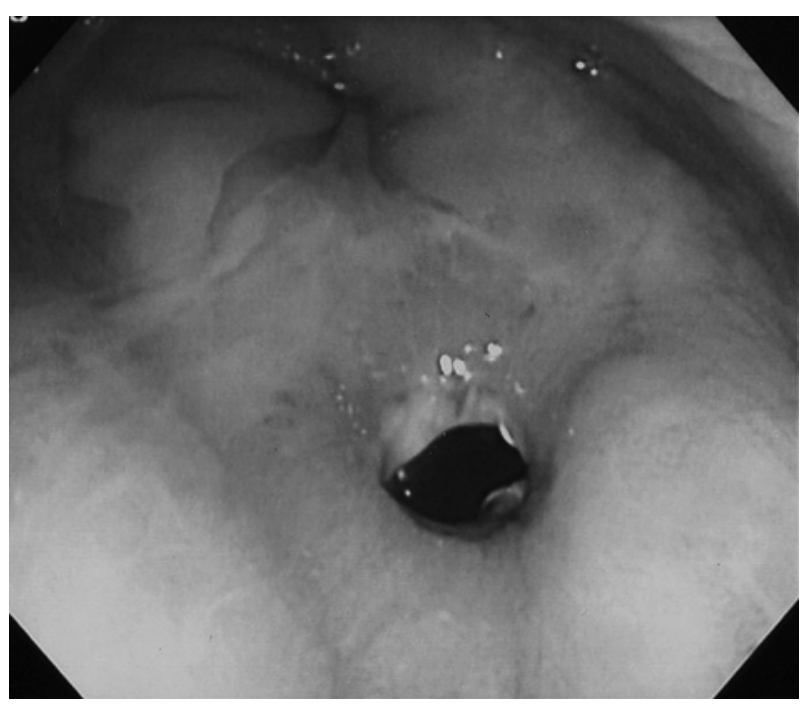




\section{References}

1 Knight CD, Griffen FD: An improved technique for low anterior resection of the rectum using the EEA stapler. Surgery 1980;88:710-714.

2 Baran JJ, Goldstein SD, Resnik AM: The double-staple technique in colorectal anastomoses: a critical review. Am Surg 1992;58:270-272.

-3 Rex JC Jr, Khubchandani IT: Rectovaginal fistula: complication of low anterior resection. Dis Colon Rectum 1992;35:354-356.

4 Nakagoe T, Sawai T, Tuji T, Nanashima A, Yamaguchi H, Yasutake T, Ayabe Y: Successful transvaginal repair of a rectovaginal fistula developing after doublestapled anastomosis in low anterior resection: report of four cases. Surg Today 1999;29:443-445.

-5 Arbman G: Rectovaginal fistulas and the double-stapling technique. Dis Colon Rectum 1993;36:310-311.

6 Sugarbaker PH: Rectovaginal fistula following low circular stapled anastomosis in women with rectal cancer. J Surg Oncol 1996;61:155-158.

7 Kosugi C, Saito N, Kimata Y, Ono M, Sugito M, Ito M, Sato K, Koda K, Miyazaki M: Rectovaginal fistulas after rectal cancer surgery: incidence and operative repair by gluteal-fold flap repair. Surgery 2005;137:329-336. 